\title{
Real-world evidence data on the monoclonal antibody erenumab in migraine prevention: perspectives of treating physicians in Germany
}

\author{
Andreas Straube ${ }^{1}$, Philipp Stude ${ }^{2}$, Charly Gaul ${ }^{3}$, Katrin Schuh ${ }^{4^{*}}$ and Mirja Koch ${ }^{5^{*}}$ (D)
}

\begin{abstract}
Background: Erenumab, the first-in-class fully human monoclonal antibody targeting the calcitonin gene-related peptide receptor, was shown to be efficacious and safe for the prophylactic treatment of migraine in adults in randomized clinical trials. Large-scale, real-world evidence in multi-centre settings is still needed to confirm these results. Erenumab patient profiles outside clinical trials and physicians' treatment patterns, as well as data from patients treated in Germany, a severely impacted population, are not published yet.

Methods: TELESCOPE was a multi-centre survey gathering real-world data from 45 German headache centres between July 2019 and December 2019. The project consisted of two parts. In the first part, treating physicians shared their experiences on current erenumab treatment with regard to patient profiles, treatment patterns and treatment responses. In the second part, a retrospective chart review was conducted of 542 migraine patients treated with erenumab for at least three months. Treatment responses focused on various aspects of patients' quality of life.

Results: The analysis of 542 patients' charts revealed that three-month treatment with erenumab significantly reduced monthly headaches, migraine and acute medication days. Furthermore, headache intensity and frequency were reduced in over $75 \%$ and accompanying aura in $35 \%$ of patients. The clinical global impression scale revealed a general improvement in $91 \%$ of patients. According to the treating physicians' professional judgement, $83 \%$ of patients responded to erenumab and $80 \%$ were satisfied with the treatment. Physicians evaluated restricted quality of life, the number of monthly migraine days and previous, prophylactic treatments as the main components of the current patient profile for monoclonal antibody recipients. Based on the assessment of physicians, erenumab reduced migraine symptoms in $65 \%$ and increased quality of life in more than $75 \%$ of their patients.
\end{abstract}

\footnotetext{
* Correspondence: Katrin.Schuh@novartis.com; Mirja.Koch@novartis.com

${ }^{4}$ Clinical Research Neuroscience, Novartis Pharma GmbH, Roonstrasse 25, 90429 Nürnberg, Germany

${ }^{5}$ Global Medical Affairs Neuroscience, Novartis Pharma AG, Fabrikstrasse 2, 4056 Basel, Switzerland

Full list of author information is available at the end of the article
}

(c) The Author(s). 2021 Open Access This article is licensed under a Creative Commons Attribution 4.0 International License, which permits use, sharing, adaptation, distribution and reproduction in any medium or format, as long as you give appropriate credit to the original author(s) and the source, provide a link to the Creative Commons licence, and indicate if changes were made. The images or other third party material in this article are included in the article's Creative Commons licence, unless indicated otherwise in a credit line to the material. If material is not included in the article's Creative Commons licence and your intended use is not permitted by statutory regulation or exceeds the permitted use, you will need to obtain permission directly from the copyright holder. To view a copy of this licence, visit http://creativecommons.org/licenses/by/4.0/. The Creative Commons Public Domain Dedication waiver (http://creativecommons.org/publicdomain/zero/1.0/) applies to the data made available in this article, unless otherwise stated in a credit line to the data. 
Conclusions: TELESCOPE confirms positive treatment responses with erenumab shown in clinical trials in a realworld multi-centre setting. The results show consistently positive experiences of physicians utilizing erenumab in clinical practice and underline that therapy with this monoclonal antibody is effective in migraine patients, particular in those, who have failed several prophylactic therapies.

Keywords: Erenumab, Prophylactic treatment, Migraine, Real-world evidence

\section{Background}

Migraine is considered one of the most prevalent, disabling conditions affecting millions of people worldwide, especially women within the age of 15-49 years [1]. A national population-based survey in Germany, conducted between October 2019 and March 2020, revealed that $14.8 \%$ of women and $6.0 \%$ of men met all of the diagnostic criteria for migraine [2]. Migraine can be classified as episodic or chronic, based on monthly headache days (MHD) and monthly migraine days (MMD); patients with fewer than 15 MHD have episodic migraine (EM) [3], and those with at least 15 MHD (and at least 8 MMD) for more than 3 months have chronic migraine (CM) [4]. Current treatment options for migraine include a combination of both acute treatment of headache attacks and prophylactic therapies. The latter is suitable for patients with more than four migraine attacks that cause disabilities for two or more days a month and cannot be controlled by the use of acute medication [5]. The majority of available prophylactic medications were originally developed for other conditions, such as hypertension, epilepsy, and depression. In epidemiological studies it was shown that the treatment with these often resulted in discontinuation already during the first 60 days due to a lack of efficacy or poor tolerability [6].

Calcitonin gene-related peptide (CGRP) has been shown to play a pivotal role in migraine pathophysiology [7]. A fundamental advance in migraine management has been the development of monoclonal antibodies targeting the CGRP pathway, representing the first selective prophylactic treatments based on the pathophysiology of migraine [8]. Erenumab, the first-in-class fully human monoclonal antibody targeting the CGRP receptor, showed efficacy and safety in large randomized clinical trials for preventive treatment of migraine patients with and without prior treatment failures [9-12]. Recently published real-world data have further confirmed the efficacy and tolerability profile of erenumab in patients suffering from migraine who failed prior prophylactic treatments [13-31]. However, these real-world publications were mainly single-centre investigations, comprised of small patient population or did not focus on the German population. Due to the requirements of the German Federal Joint Committee (Gemeinsamer Bundesausschuss = GBA) for reimbursement, mainly severely affected patients are treated so far, the prerequisites being the failure of at least five approved prophylactics or the contraindications to those. In CM patients, prior failure to onabotulinumtoxin $\mathrm{A}$ is additionally required [32]. This unique situation in Germany provides a particularly, severely affected patient population in clinical practice, which is not sufficiently investigated so far. Furthermore, direct experience from the treating physicians on patient profiles and treatment patterns of erenumab in daily routines have not been reported so far.

In TELESCOPE, the first real-world data was collected in a multi-centre setting across Germany in order to characterize the use of erenumab in clinical practice from the treating physicians' perspective. An online survey was conducted to document physicians' experiences regarding current patient profiles for CGRP antibody recipients, therapy approach and treatment responses with erenumab. A retrospective chart review was conducted to analyse data of migraine patients being treated with erenumab for at least three months, focusing on treatment effects and various aspects of patients' quality of life (QoL).

\section{Methods}

TELESCOPE was a German online survey, capturing the professional assessment of erenumab treatment from 45 headache specialists. The survey was accomplished between July 2019 and December 2019. The project consisted of two parts. In the first part, treating physicians reported experiences on treating patients with erenumab. In the second part, physicians consecutively documented at least 10 but not more than 30 of their patients that had received erenumab for $\geq 3$ month via a retrospective chart review. A steering committee comprising of medical and scientific experts in neurology and migraine was established to provide independent advice and input into the development of the survey questionnaire. The participating physicians completed the 24 items of the standardized questionnaire and were asked to respond, supported by a comprehensive research of their patient files.

For the first part of the questionnaire, participating physicians shared their experience with erenumab (including their current therapy approach and treatment responses. The first four questions reflected the characteristics of the participating centres (e.g. number of 
headache and migraine patients treated). The following four questions evaluated physicians' experiences in treating migraine patients; i.e. the current patient profile for the initiation of monoclonal antibody treatment, treatment management and general outcome of the treatment with erenumab. The latter included onset of treatment effect, reduction of MMD and headache intensity, improvement in QoL and improvement of typical migraine symptoms (e.g. sensitivity for light and noise, nausea/emesis, allodynia, and aura). Another question concerned physicians' opinion on the most important criteria for therapy outcome evaluation. The options available were reduction of MMD by at least $30 \%$ or $50 \%$ reduction of headache intensity, change in QoL and reduction of typical migraine symptoms.

The remaining 15 questions of the questionnaire reflected the retrospective chart review. Individual patients' demographics and treatment characteristics were collected anonymised including gender, diagnosis of EM and $\mathrm{CM}$, health insurance status, number and type of previous, prophylactic, medical and non-medical treatments and data on erenumab treatment (current doses and adjustments). The survey also inquired the physicians' opinion on patients' satisfaction and treatment success. For the latter, physicians were asked how many patients they consider as "responders" according to their professional judgement, which was based on the reduction of headache days and the overall impression of their patients in order to reflect current treatment routine in Germany. The last 8 questions concentrated on migraine-specific parameters (MMD, MHD and monthly acute medication days, attack intensity, frequency and accompanying aura), patients' global impression on disease improvement and migraine-related symptoms. The occurrence of accompanying aura symptoms was defined by the treating physicians.

To avoid a selection bias, physicians were instructed to collect retrospective data of a consecutive series of patients diagnosed with EM or CM. Data were handled confidentially and anonymity of patients was secured. Due to the research format, this study was exempted from ethics committee review. All data on physicians' opinion and patient data were analysed descriptively. If needed for certain explorative analyses, the pairedsamples t-test for continuous data were applied. $P$-values $<0.05$ were considered statistically significant.

\section{Results}

\section{Treating physicians' perspectives}

Headache specialists of 45 German headache centres participated in the online survey by completing a questionnaire and a comprehensive, retrospective chart review. At all sites, participating physicians had been treating 202 migraine patients in average during the previous three months; 12 sites $(27 \%)$ reported treating more than 300 migraine patients. In total, 9099 migraine patients have been treated by all participating physicians within the preceding three months, and of these, in average $19.1 \%$ with monoclonal antibodies. Thereof, $78.6 \%$ had erenumab for at least three months.

The current patient profile for treatment initiation was evaluated according to professional assessments of participating physicians. The four main reasons (rated as 'very important' and 'important') for treatment initiation with monoclonal antibodies were a restricted QoL $(100 \% ; n=45)$, number of MMD (98\%; $n=44)$, number of previously tried prophylactic therapies $(89 \%$; $n=40)$ and monthly days of acute medication use (87\%; $n=39$ ). Missed days at work/school were considered as important by half ( $51 \% ; n=23)$ and comorbidities by a third (33\%; $n=15)$ of the physicians (Fig. 1).

Physicians' file research of the total population, who have been treated for at least three months with erenumab revealed that the majority of patients $(84 \%)$ initiated treatment with $70 \mathrm{mg}$, whereas for $16 \%$ the higher dose of $140 \mathrm{mg}$ was chosen. They reported an onset of effect for $70 \% \pm 24 \%$ of the population after the first injection, $20 \% \pm 15 \%$ after the second and $8 \% \pm 10 \%$ after the third antibody injection. In case of nonresponse by physicians' discretion, $70 \%$ of the physicians discontinued monoclonal antibodies after 3 consecutive months of treatment (data not shown). According to the experience of treating physicians, erenumab treatment had led to a relevant reduction in headache intensity in $77 \%$ of the population and patient-relevant improvement of QoL in $76 \%$. During the data collection period in 2019, an at least $30 \%$-reduction in MMD was achieved in $73 \%$ and a $50 \%$-reduction in $66 \%$ of patients (Fig. 2). Nearly three quarters of the participating physicians $(73 \%)$ considered a reduction of MMD by $50 \%$ and $30 \%$ the most important criteria to evaluate treatment response in clinical practice. Further criteria were patient-relevant improvements of QoL $(16 \%)$ and reduction of headache intensity (4\%) (data not shown).

\section{Medical chart review of erenumab treated patients}

In the second part of TELESCOPE, medical chart data of 542 consecutively selected erenumab patients were gathered anonymously. According to the retrospective analysis of the medical records, $85 \%$ of the patients were female, $50 \%$ were suffering from EM and $50 \%$ from CM. About half of the patients (54\%) currently received an erenumab dose of $70 \mathrm{mg}$ and $46 \%$ the higher dose of $140 \mathrm{mg}$. Patients diagnosed with CM more frequently obtained the higher dose of $140 \mathrm{mg}$ erenumab than patients with EM ( $58 \%$ vs. $35 \%$ ). Throughout the previous treatment period, $48.2 \%$ of all patients received a dose adjustment, which was more often applied to CM 


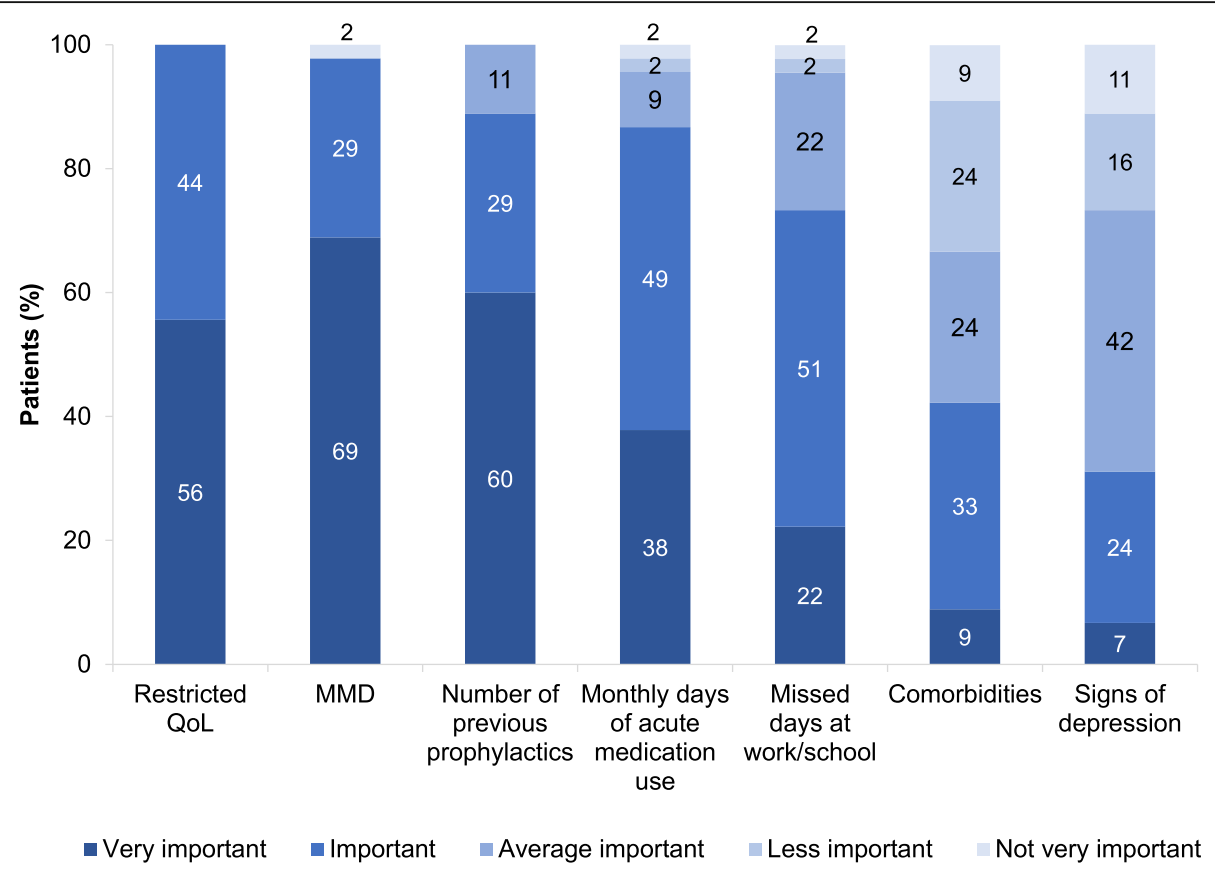

Fig. 1 Reasons for initiation of treatment with a monoclonal antibody - physicians' perspective. $N=45$ (number of physicians); QoL=quality of life; $\mathrm{MMD}=$ =monthly migraine days; Seven distinct reasons for the initiation of treatment with a monoclonal antibody were evaluated by the treating physician according to the level of importance

patients than to EM patients ( $57 \%$ vs. $40 \%$ ). In $94 \%$ of the patients who started with $70 \mathrm{mg}(N=252)$, the dose of erenumab was escalated from $70 \mathrm{mg}$ to $140 \mathrm{mg}$. In some patients $(8.3 \%)$, who started treatment with $140 \mathrm{mg}(N=290)$, the dose was de-escalated to $70 \mathrm{mg}$ (data not shown). At the time Telescope was conducted, patients have been treated with erenumab for $6.9 \pm 2.7$ months in average (Table 1).

\section{Previous migraine treatments}

All patients $(100 \%)$ tried or were not suitable for in average $4.6 \pm 1.4$ prior medical prophylactic therapies,

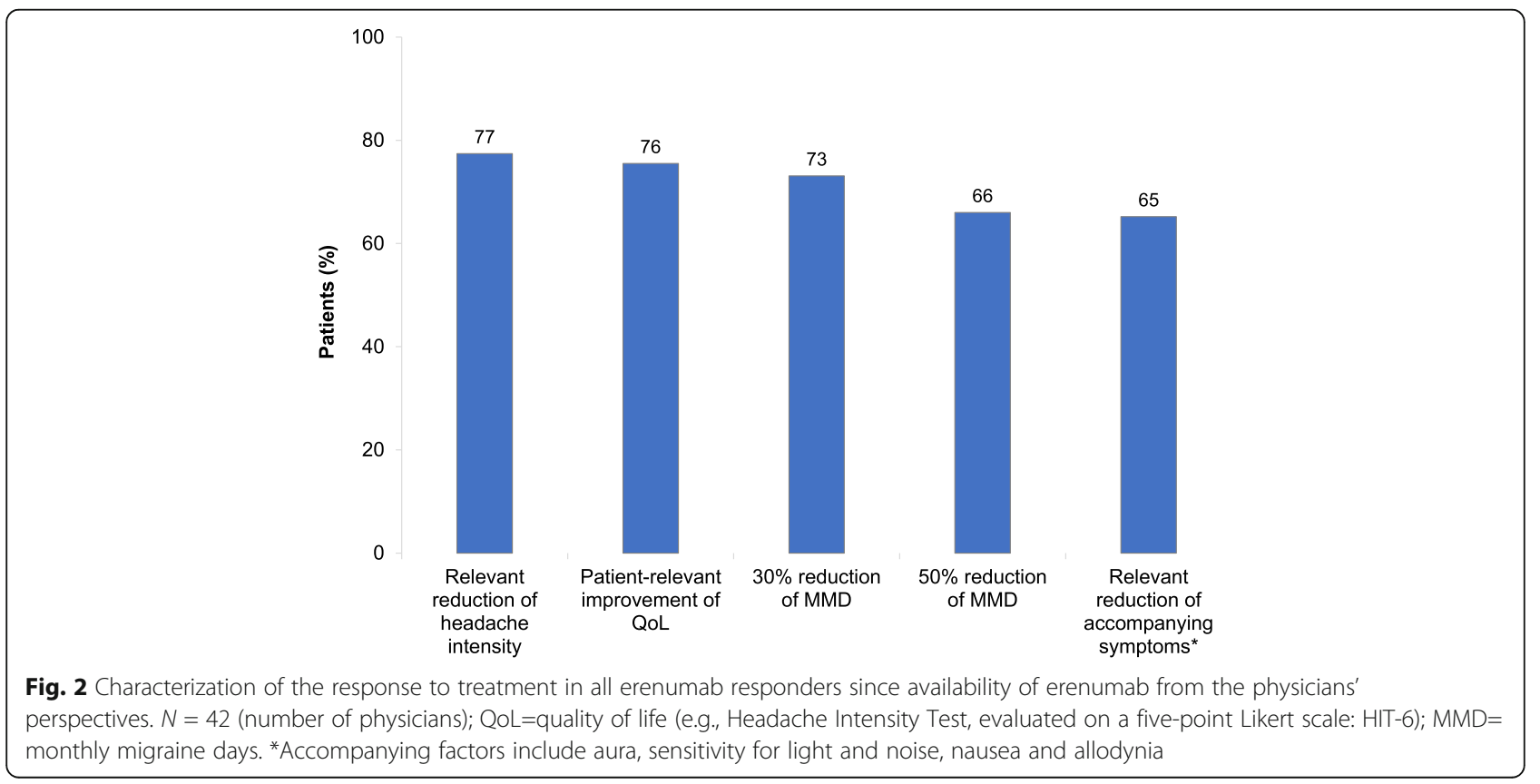


Table 1 Patient characteristics

\begin{tabular}{ll}
\hline & $\begin{array}{l}\text { Patients } \\
\mathbf{N}=\mathbf{5 4 2}\end{array}$ \\
\hline Sex, n (\%) & $81(15)$ \\
Male & $461(85)$ \\
Female & \\
Migraine type, $\mathrm{n}(\%)$ & $271(50)$ \\
Chronic migraine & $271(50)$ \\
Episodic migraine & \\
Current dose of erenumab, $\mathrm{n}(\%)$ & $290(54)$ \\
70 mg & $252(46)$ \\
140 mg & \\
Insurance status, $\mathrm{n}$ (\%) & $474(87)$ \\
Statutory health insurance & $68(13)$ \\
Private health insurance & $6.9(2.7)$
\end{tabular}

$S D$ standard deviation

before initiating the treatment with erenumab. Previous treatments included mostly topiramate in $85.1 \%$ of the patients, beta blocker in $80.6 \%$, amitriptyline in $72.0 \%$ and flunarizine in $49.8 \%$. Onabotulinumtoxin $\mathrm{A}$ was additionally tried by 204 patients, all of whom were diagnosed with CM. Hence, $75.3 \%$ of CM patients also failed previous treatment with onabotulinumtoxin $\mathrm{A}$. The majority of all patients $(74.9 \%)$ also used non-medical therapies as prophylactic treatments with relaxation therapies being the most frequently used (Table 2).

\section{Effects of erenumab treatment}

After a three-month erenumab treatment period, MHD, MMD, as well as monthly acute medication days were significantly reduced in documented patients. Compared to baseline, the mean reduction in MHD after 3 months was -7.5 days (from $14.9 \pm 6.6$ to $7.4 \pm 6.2 ; P<0.0001$ ). The mean reduction in MMD was -6.2 days after 3 month (from $12.1 \pm 5.9$ to $5.9 \pm 5.5 ; P<0.0001$ ) and in monthly acute medication days -6.4 days (from $11.5 \pm$ 5.8 to $5.1 \pm 4.8, P<0.0001$ ) (Fig. 3 ). Of 542 migraine patients, $82.7 \%$ were considered as "responders", according to physicians' professional judgement, which was based on the reduction of headache days and the overall impression of their patients. Treating physicians reported that $79.5 \%$ of all patients were satisfied with erenumab treatment. Very high "responder" $(88.6 \%)$ and patient's satisfaction $(85.2 \%)$ were evaluated in patients with EM (Table 3).

Based on the clinical global impression scale, treatment with erenumab for at least three months led to a general improvement (very much, clearly and somewhat improved) in $91 \%$ of all patients. Patients with EM showed somewhat better improvements compared to those with CM; (very much $(38.4 \%$ vs. $33.9 \%$ ) and clearly improved (41.1\% vs. $31.4 \%$ ). Less than $0.5 \%$ of all patients stated a deterioration of the disease during erenumab treatment (Fig. 4). Furthermore, relevant improvements in migraine-specific headache parameters and migraine-related symptoms were reported. In detail, $75.3 \%$ of all patients had reduced headache intensity, $85.4 \%$ reduced frequency of headaches and $35.2 \%$ accompanying aura after three months of erenumab treatment. The presence of aura at baseline was not investigated. Improvements of attack intensity and frequency, were more pronounced in EM patients compared to patients with CM $(81.2 \%$ vs. $69.4 \%$ and $89.7 \%$ vs. $81.2 \%$ ). A reduction of accompanying aura was also an effect rather seen in EM patients than in CM patients (41.7\% vs. $28.8 \%$ ) (Fig. 5).

Regarding migraine-related symptoms, physicians reported that nausea and emesis were reduced in $46.7 \%$ of all patients and sensitivity for light and noise in $42.4 \%$ of patients three months after initial treatment with erenumab. About $39.9 \%$ of patients were uncertain regarding improvement of these symptoms. The effects of erenumab on migraine-related symptoms were similar in both, EM and CM patients (Fig. 6).

\section{Discussion}

TELESCOPE provides us with real-world evidence data on erenumab treatment gathered in a large German cohort. Three-month treatment with erenumab significantly decreased MMD, MHD and acute medication days, reduced headache frequency, intensity and frequency of accompanying aura, improved the patients' clinical global impression scale and QoL in a patient population with several treatment failures. A reduction in nausea/emesis, light/noise sensitivity and the need to rest was reported in a remarkable number of patients. In addition, headache specialists reported the current patient profile and specific treatment patterns when prescribing erenumab. These findings captured the physicians' professional perspective and judgement on the initial treatment outcome after three months of erenumab treatment in routine clinical practice and will thus be a valuable complement to the data obtained from randomized clinical trials. Furthermore, our data offer valuable information for experienced clinicians and might additionally provide guidance for healthcare specialists who have limited clinical experience with monoclonal antibodies in migraine management.

Treating physicians retrospectively documented data of 542 migraine patients. The vast majority of this cohort were members of a statutory $(87 \%)$ health insurance, while the remaining $13 \%$ contributed to a private health insurance. This broadly reflects the current distribution of the German health care system with $10.5 \%$ 
Table 2 Previous prophylactic therapies of erenumab patients

\begin{tabular}{|c|c|c|}
\hline Medical therapies $^{a}$ & $\begin{array}{l}\text { Previous therapy } \\
\text { N (\%) }\end{array}$ & $\begin{array}{l}\text { Contraindication against therapy } \\
\mathrm{N}(\%)\end{array}$ \\
\hline Topiramate & $461(85.1)$ & $41(7.6)$ \\
\hline Beta blocker & $437(80.6)$ & $68(12.5)$ \\
\hline Amitriptyline & $390(72.0)$ & $81(14.9)$ \\
\hline Flunarizine & $270(49.8)$ & $167(30.8)$ \\
\hline Onabotulinumtoxin $A^{b}$ & $204(37.6)$ & $8(1.5)$ \\
\hline Valproate & $107(19.7)$ & $249(45.9)$ \\
\hline Others & $406(74.9)$ & - \\
\hline No previous therapy & $9(1.7)$ & \\
\hline No contraindications & - & $217(40.0)$ \\
\hline Number of previous therapies, mean (SD) & $3.4(1.4)$ & \\
\hline Number of previous therapies and contraindications, mean (SD) & $4.6(1.4)$ & \\
\hline Non-medical therapies & & N (\%) \\
\hline Yes & & $406(74.9)$ \\
\hline No & & $136(25.1)$ \\
\hline Relaxation therapies & & $338(62.4)$ \\
\hline Physical exercise (sports) & & $270(49.8)$ \\
\hline Acupuncture & & $219(40.4)$ \\
\hline Psychological therapy of pain & & $168(31.0)$ \\
\hline
\end{tabular}

$S D$ standard deviation, $N 542$ (number of patients)

${ }^{a}$ Multiple responses were possible

${ }^{b}$ Only patients with chronic migraine additionally received onabotulinum A (i.e. $75.3 \%$ of CM patients)

privately insured citizens [33]. Patients failed or had contraindications to an average of five prior, prophylactic migraine treatments before erenumab was prescribed. The most common discontinued prophylactic therapies were topiramate, beta blocker and amitriptyline. Three quarters of $\mathrm{CM}$ patients additional received onabotulinumtoxin A. This high number of treatment failures can be attributed to the regulations of the German GBA for the reimbursement of CGRP monoclonal antibodies by the statuary health systems and thus mirrors the current highly impacted patient population that receives erenumab treatment in Germany [32]. The data confirm that physicians follow the requirements of the statuary health system when prescribing monoclonal antibodies. Furthermore, as recommended by current guidelines, the majority of patients $(75 \%)$ also applied non-medical therapies for migraine prevention with relaxation techniques being used most frequently [34].

Three months treatment with erenumab led to a significant reduction of -6.2 MMD, -7.5 MHD, and -6.4 monthly acute medication days in this analysis. Data investigating erenumab treatment in large numbers of both $\mathrm{CM}$ and EM patients in real-world multi-centre settings are limited. However, our results are in the range of existing real-world evidence with erenumab gathered mainly in single-centre settings. Mean reductions in MMD of -3 to -13 days, MHD of -3 to -10 days and of -3 to -6 monthly acute medication days after three-month erenumab treatment in patients with an average of four up to eight prior prophylactic treatment failures were reported [13-20, 22-25]. All real-life data published so far complement and partly exceed the effects shown in clinical trials $[8-10,12]$.

Results of our analysis comprising of a substantial number of multiple treatment-refractory EM patients in particular support findings of the LIBERTY trial, in which a significant reduction of MMD and acute medication days was demonstrated. Under the treatment of erenumab, $30 \%$ of all patients reached a $\geq 50 \%$ reduction in MMD, compared to $14 \%$ in the placebo group [12]. In our survey, participating headache specialists and neurologists considered $83 \%$ of all patients as "responders" (89\% of EM and $77 \%$ of CM patients). The estimation of the percentage of "responders was according to the physicians' professional judgement, which was based on the reduction of headache days and the overall impression of the patients in order to reflect current treatment routine in Germany. Of note, only patients that actually had received 3 injections of erenumab over three months were analysed. Hence, patients who discontinued therapy early were not included in the "responder evaluation". Even though current guidelines 


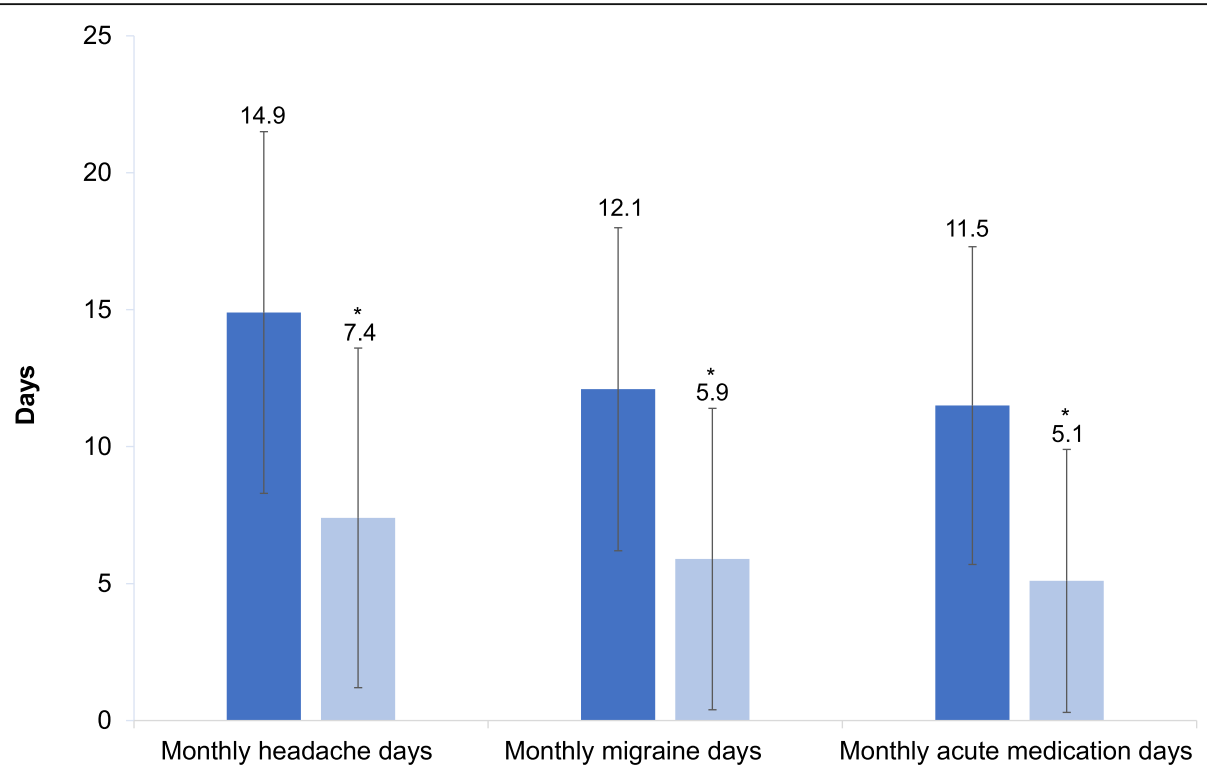

- Prior to erenumab treatment After 3 month of erenumab treatment

Fig. 3 Headache-, migraine-, and medication days after 3 months of erenumab treatment - medical chart review ( $N=542)$; MHD = monthly headache days; $\mathrm{MMD}=$ monthly migraine days; ${ }^{*} P<0.0001$

[35] recommend evaluation of the response to treatment with monoclonal antibodies only after three months, there may be reasons for early discontinuation, such as patients wish.

According to the chart review, $54 \%$ of all patients currently received $70 \mathrm{mg}$ of erenumab and $46 \%$ the higher dose of $140 \mathrm{mg}$. CM patients more frequently obtained the higher dose of $140 \mathrm{mg}$ than EM patients (58\% vs. $35 \%)$. Physicians reported that in $84 \%$ of the erenumabtreated population, treatment is usually started with the lower dose of $70 \mathrm{mg}$. This was reflected by the medical chart review as $94 \%$ of patients who currently received

Table 3 "Responder" and patients' satisfaction - physicians' judgement

\begin{tabular}{|c|c|c|c|}
\hline & $\begin{array}{l}\text { Overall } \\
N=542\end{array}$ & $\begin{array}{l}E M \\
N=271\end{array}$ & $\begin{array}{l}C M \\
N=271\end{array}$ \\
\hline \multicolumn{4}{|c|}{ "Responder", n (\%) } \\
\hline Yes & $448(82.7)$ & $240(88.6)$ & $208(76.8)$ \\
\hline No & $94(17.3)$ & $31(11.4)$ & $63(23.2)$ \\
\hline \multicolumn{4}{|c|}{ Patient satisfaction, n (\%) } \\
\hline Yes & $431(79.5)$ & $231(85.2)$ & $200(73.8)$ \\
\hline No & $73(13.5)$ & $26(9.6)$ & $47(17.3)$ \\
\hline Uncertain & $38(7)$ & $14(5.2)$ & $24(8.9)$ \\
\hline
\end{tabular}

Treating physicians classified "responder" according to their professional judgement, which was based on the reduction of headache days and the overall impression of their patients

$E M$ episodic migraine, $C M$ chronic migraine
$140 \mathrm{mg}(N=252)$ underwent a previous dose escalation from $70 \mathrm{mg}$ to $140 \mathrm{mg}$. TELESCOPE was conducted approximately one year after marketing authorization in the European Union (July 2018) and market launch in Germany (November 2018). Thus, physicians might have been hesitant to start erenumab treatment with the higher dose. Available evidence suggests the preferred use of a $140 \mathrm{mg}$ dose of erenumab over the $70 \mathrm{mg}$ in EM and CM patients after several prior treatment failures [36, 37]. Our data and available evidence support that severely impacted patients with high numbers of prophylactic treatment failures and high numbers of MMD at baseline may warrant the higher dosage in order to obtain the full impact of erenumab. Further real-life studies could show whether the dose preference will change over time and experience with erenumab.

The physicians specified restricted QoL, high numbers of MMD and the number of previous, unsuccessful prophylactic treatments as the patient profile suitable for treatment initiation with monoclonal antibodies. This is in line with current German guidelines and reimbursement restrictions [32, 35]. Based on physicians' clinical experience, erenumab treatment mediated a relevant reduction in headache intensity and patient-relevant improvement of QoL in more than three quarters of the treated population. The majority of the population (70\%) experienced treatment effects already after the first injection of erenumab. This early onset of erenumab 


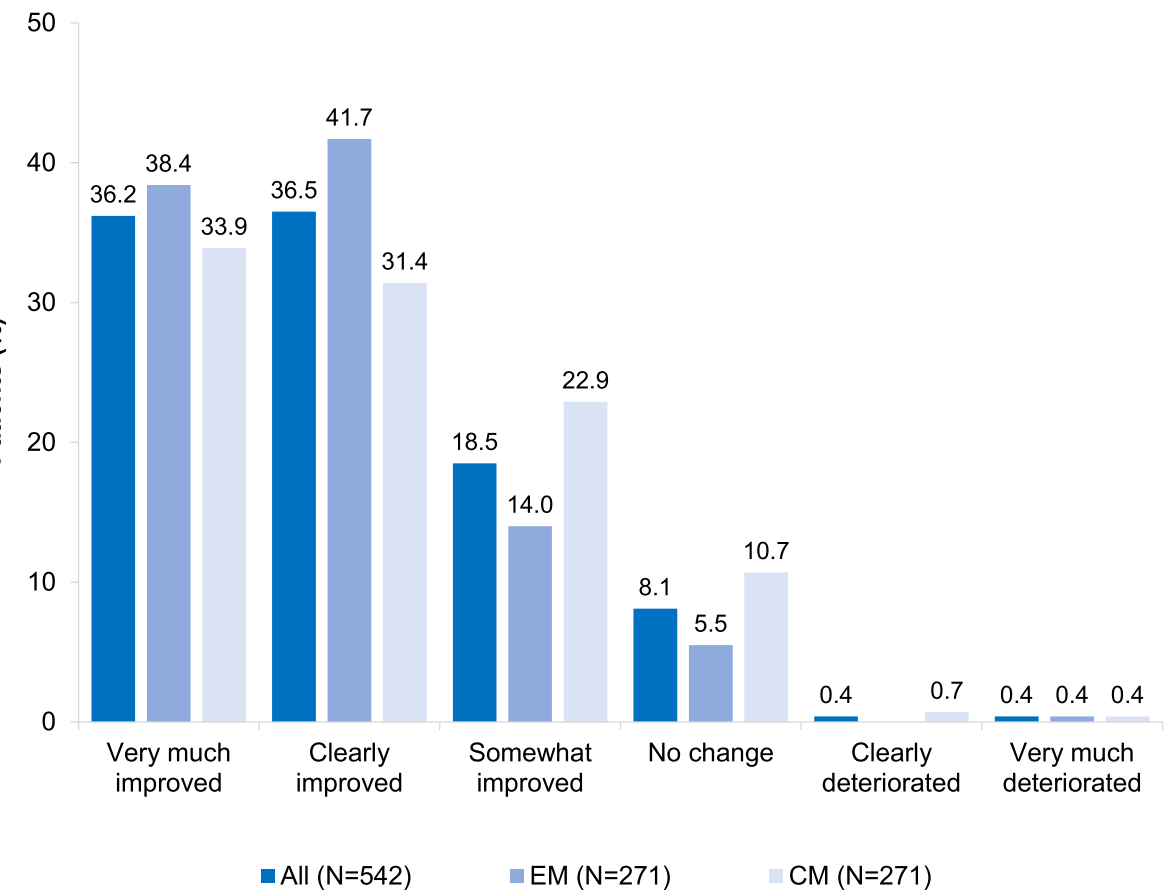

Fig. 4 Global impression scale in migraine patients after 3 months of erenumab treatment - medical chart review $(N=542)$; EM = episodic migraine; $\mathrm{CM}=$ chronic migraine; Clinical global impression scale of improvement was defined by responses of very much improved, clearly improved, somewhat improved, no change, clearly deteriorated and very much deteriorated

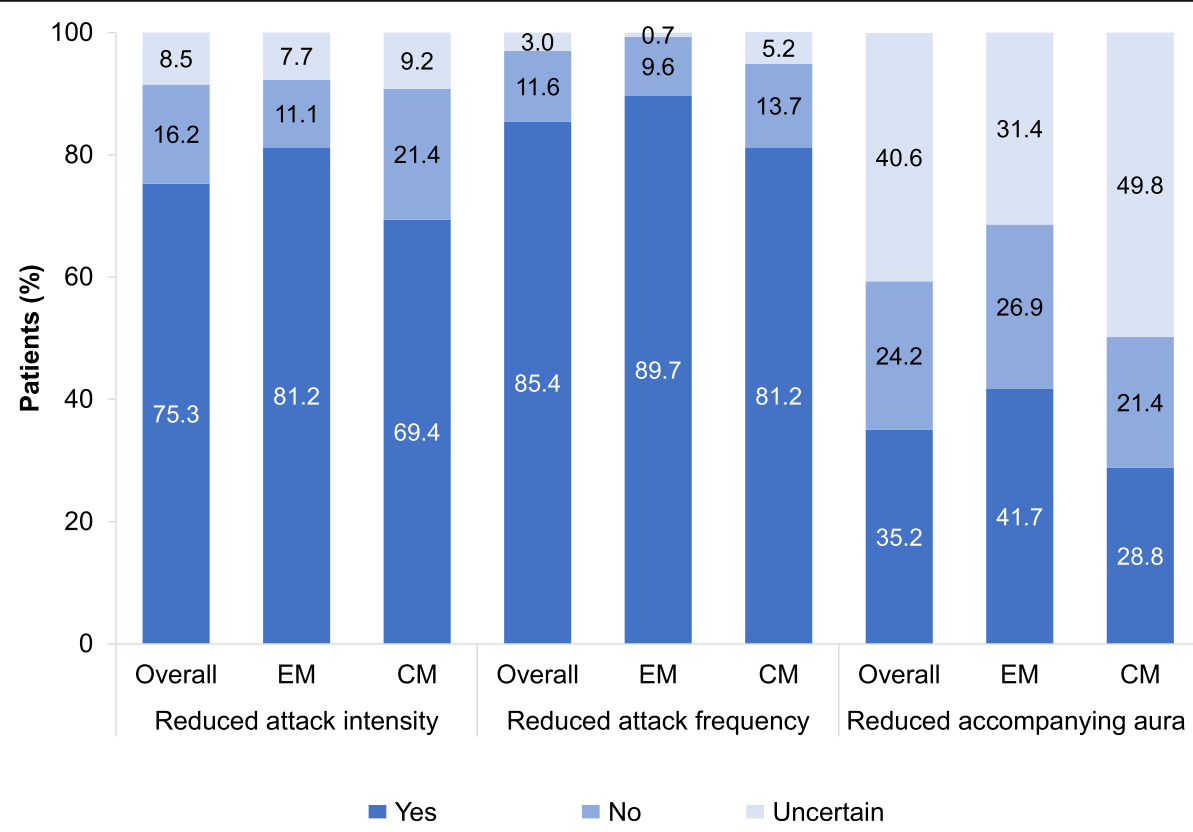

Fig. 5 Effect of erenumab on migraine-specific headache parameters after 3 months of treatment - medical chart review $(N=542)$; $E M=e p i s o d i c$ migraine $(N=271) ; C M=$ chronic migraine $(N=271)$ 


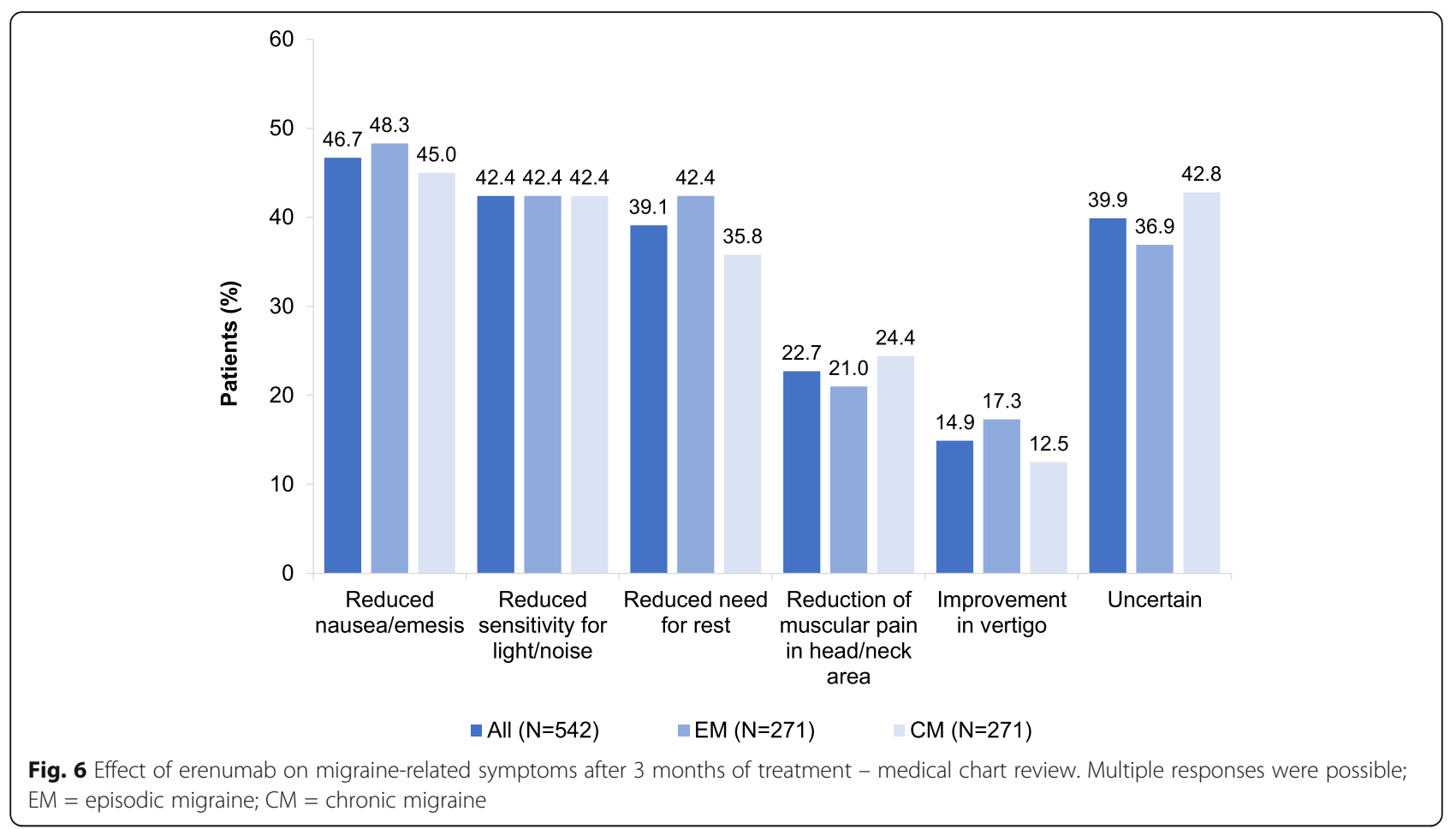

action within the first weeks of treatment confirms data retrieved from controlled studies for CM and EM patients [38].

Besides the $50 \%$ and $30 \%$ reduction in MMD, physicians rated the importance of patient-relevant improvements of QoL, as one of the most important criteria to evaluate treatment responses in clinical practice. Erenumab-mediated improvements in QoL were reported in clinical practice, mainly using the Headache Impact Test (HIT) -6 score $[13,14,16,17,19,21,22,26]$.

The impact of migraine on QoL is often evaluated through the analysis of headache-specific validated questionnaires, in which other aspects of QoL are insufficiently covered. Complementary information, such as migraine-specific headache parameter, global impression, patients' satisfaction and symptoms might better reflect the overall treatment benefit. According to the medical chart review, general improvements based on patients' global assessment scale was seen in almost all patients (91\%). Furthermore, $85 \%$ of EM patients and $74 \%$ of $\mathrm{CM}$ patients were satisfied with erenumab treatment. This is in line with findings of another German real-life cohort in which the majority of patients ( $82 \%)$ also reported to be satisfied with erenumab treatment [18]. Besides treatment efficacy, treatment satisfaction has been associated with drug adherence [39] and may be particularly important in therapy-refractory migraine patients as presented here. Another relevant therapeutic effect of erenumab treatment was evaluated by the reduction of attack intensity and frequency in over $80 \%$ of EM and the majority of $\mathrm{CM}$ patients. In addition, patients reported reduction of accompanying aura ( $42 \%$ of EM and $29 \%$ of CM patients). These results are in line with the treating physicians' experience reported within the first part of our survey, who stated that $77 \%$ of their patients experience a relevant reduction of headache intensity and $65 \%$ in accompanying symptoms, such as aura. In clinical practice, positive effects of treatment with erenumab on headache intensity have been shown mainly in CM patients [16, 18, 19]. With our data, we can support the existing real-world evidence in $\mathrm{CM}$ patients in a large cohort and extend it to EM patients.

To date, there are no well-proven treatments available to reduce accompanying aura. An aura prevalence of $35 \%-52 \%$ [10-12] and $23 \%-35 \%[15,17,30,31]$ was reported in clinical trials and in real-world studies investigating erenumab, respectively. In our survey, physicians reported that $35 \%$ of their patients had reduced accompanying aura, which was more pronounced in EM than in CM patients ( $41.7 \%$ and $28.8 \%$ ). Interestingly, this is line with recent observations reported by Ament et al. 2021 for the treatment with another CGRP-monoclonal antibody [40]. Due to our survey design, baseline values of patients with aura for comparison are not available. Further data will be needed to understand the ratio between patients with aura at baseline and patients with a relief of these symptoms, and to understand the extent of the effect. 
In the large global My Migraine Voice survey investigating disease burden of over 10,000 treatmentrefractory migraine patients, symptoms beyond the headache phase were reported by the majority of patients. In detail, over three quarters of the investigated patients were suffering additionally from light sensitivity, sound sensitivity and nausea [41]. The authors suggested an unmet need of treatments for these symptoms. In our retrospective observation erenumab treatment addressed this need in a remarkable proportion of patients. In about half of the patients, nausea or emesis and in approximately $40 \%$ sensitivity for light, noise and need for rest was reduced during erenumab treatment. Due to the retrospective character of the study an evaluation of the reduction was not feasible. But the question remains, how to accurately capture effects on non-pain symptoms in retrospective and prospective settings. Similar, albeit smaller, improvements in migraine symptoms with erenumab treatment were reported in another German real-life cohort [18]. Previous randomised trials did not focus on migraine symptoms; however, the ongoing EMBRACE study was designed to fully capture the effect of erenumab beyond MMD, on migraine-related symptoms. This study will provide further insights on the effect of erenumab treatment on non-pain symptoms and the quantification of the relief.

Some limitations need to be considered. Only specialized physicians participated in the survey implying a possible bias of the study population. The analysed population might differ from what other neurologists and general care specialists encounter in their daily routine. Patients at specialized centres are more likely to be refractory to therapies, as is reflected by the exhaustion of prior therapies and the high proportion of nonmedical procedures. On the contrary, this can also be regarded as a strength, because especially in multiple refractory patients, physicians estimated the treatment as successful and well tolerated. Further limitations are the retrospective design and the type of reporting. Collected data are mainly based on patients' medical charts and physicians' opinion. Assessments of migraine-specific headache parameter, patients' satisfaction, global impression and symptoms in real-world settings have been based on the patients' perspective gathered by the treating physician. Thus, our findings may be affected by reporting and recall bias. The somewhat better treatment effects reported here, compared to randomized trials and other real-life studies might be explained by the high expectations regarding the effectiveness of the drug and the fast onset of erenumab, which makes a treatment response in patients apparent. But, similar observations were also reported for the efficacy of triptans in real-world setting, compared to the data from randomized trials [42]. Despite these limitations, TELESCOPE provides an approach that captures the effects of erenumab in migraine patients in clinical practice and supports treatment decisions.

\section{Conclusions}

In TELESCOPE, we provide comprehensive retrospective real-world evidence in a large migraine cohort in Germany and in addition first real-life insights on physicians' overall experiences with erenumab treatment in migraine patients. A restricted QoL, number of MMD and previous prophylactic treatments represent the main components of the current patient profile for CGRP monoclonal antibody recipients. Based on the assessment of physicians, erenumab reduced migraine symptoms in $65 \%$ and increased quality of life in more than $75 \%$ of their treated population. These reports were reflected by a medical chart review of 542 refractory migraine patients. The retrospective analysis revealed that three months of erenumab treatment significantly reduced migraine-specific headache parameter and increased QoL in this severely impacted patient population. According to the treating physicians' professional judgement, the vast majority of their patients did benefit from and were satisfied with the treatment. The results of this survey show positive experiences of treating physicians with the use of erenumab in everyday clinical practice.

\section{Abbreviations \\ CGRP: Calcitonin gene-related peptide receptor; CM: Chronic migraine; EM: Episodic migraine; HIT-6: Headache Impact Test; MMD: Monthly migraine days; MHD: Monthly headache days; SD: Standard deviation; QoL: Quality of life}

\section{Acknowledgements}

The authors would like to thank the participating physicians for their support of this survey. Writing support was provided by Dr. Angelika Schedel of Schedel Medical Communication located in Nuremberg, Germany. This support included the development of all drafts of the manuscript, under the guidance of the authors, and coordination of author comments and approval, and was funded by Novartis Pharma GmbH, Nuremberg.

\section{Authors' contributions}

AS, PS, CG contributed to data acquisition, analyses, interpretation and manuscript writing. KS and MK contributed to study conception, design, data analysis and interpretation and manuscript writing. The author(s) read and approved the final manuscript.

\section{Funding}

This study was funded by Novartis Pharma GmbH, Nuremberg.

\section{Availability of data and materials}

The datasets used and analysed during the current study are available from the corresponding author on reasonable request.

\section{Declarations}

Ethics approval and consent to participate

Due to the research format, this study was exempted from ethics committee review.

Consent for publication

Not applicable. 


\section{Competing interests}

AS received honoraria for educational talks and advisory boards from Allergan Lilly, Novartis, Sanofi and Teva.

PS received honoraria for advisory boards from Novartis, TEVA, Lilly and Hormosan

CG received honoraria for consulting and lectures within the past three years from Allergan Pharma, Lilly, Novartis, Hormosan, Grünenthal, Sanofi-Aventis, Weber \& Weber, Lundbeck and TEVA. He does not hold any stocks of pharmaceutical companies. He is honorary secretary of the German Migraine and Headache Society.

KS is employed at Novartis Pharma GmbH, Nuremberg, Germany, the sponsor.

MK is employed at Novartis Pharma AG, Basel, Switzerland, the sponsor.

\section{Author details}

'Department of Neurology, University Hospital LMU,

Ludwig-Maximillians-University, 81377 Munich, Germany. ${ }^{2}$ Neurological Practice Dr. Stude, Bochum, Germany. ${ }^{3}$ Headache Center Frankfurt, Frankfurt a. Main, Germany. ${ }^{4}$ Clinical Research Neuroscience, Novartis Pharma GmbH, Roonstrasse 25, 90429 Nürnberg, Germany. ${ }^{5}$ Global Medical Affairs Neuroscience, Novartis Pharma AG, Fabrikstrasse 2, 4056 Basel, Switzerland.

\section{Received: 16 June 2021 Accepted: 21 October 2021}

Published online: 06 November 2021

\section{References}

1. GBD 2016 Headache Collaborators (2018) Global, regional, and national burden of migraine and tension-type headache, 1990-2016: a systematic analysis for the Global Burden of Disease Study 2016. Lancet Neurol 17(11): 954-976. https://doi.org/10.1016/s1474-4422(18)30322-3

2. Porst $M$, Wengler A, Leddin J, Neuhäuser H, Katsarava Z, von der Lippe E et al (2020) Migräne und Spannungskopfschmerz in Deutschland. Prävalenz und Erkrankungsschwere im Rahmen der Krankheitslast-Studie BURDEN 2020. J Health Monit 5(Special Issue 6). https://doi.org/10.25646/6990

3. Goadsby PJ, Evers S (2020) International classification of headache disorders - ICHD-4 alpha. Cephalalgia 40(9):887-888. https://doi.org/10.1177/03331 02420919098

4. Headache Classification Committee of the International Headache Society (IHS) (2018) The international classification of headache disorders, 3rd edition. Cephalalgia 38(1):1-211. https://doi.org/10.1177/0333102417738202

5. Steiner TJ, Jensen R, Katsarava Z, Linde M, MacGregor EA, Osipova V et al (2019) Aids to management of headache disorders in primary care (2nd edition): on behalf of the European Headache Federation and Lifting The Burden: the Global Campaign against Headache. J Headache Pain 20(1):57. https://doi.org/10.1186/s10194-018-0899-2

6. Hepp Z, Dodick DW, Varon SF, Chia J, Matthew N, Gillard P et al (2017) Persistence and switching patterns of oral migraine prophylactic medications among patients with chronic migraine: a retrospective claims analysis. Cephalalgia 37(5):470-485. https://doi.org/10.1177/03331024166783 82

7. Edvinsson L, Mulder H, Goadsby PJ, Uddman R (1998) Calcitonin generelated peptide and nitric oxide in the trigeminal ganglion: cerebral vasodilatation from trigeminal nerve stimulation involves mainly calcitonin gene-related peptide. J Auton Nerv Syst 70(1-2):15-22. https://doi.org/10.1 016/s0165-1838(98)00033-2

8. Tepper SJ (2019) CGRP and headache: a brief review. Neurol Sci 40(Suppl 1): 99-105. https://doi.org/10.1007/s10072-019-03769-8

9. Dodick DW, Ashina M, Brandes JL, Kudrow D, Lanteri-Minet M, Osipova V et al (2018) ARISE: a phase 3 randomized trial of erenumab for episodic migraine. Cephalalgia 38(6):1026-1037. https://doi.org/10.1177/033310241 8759786

10. Goadsby PJ, Reuter U, Hallström Y, Broessner G, Bonner JH, Zhang F et al (2017) A controlled trial of erenumab for episodic migraine. N Engl J Med 377(22):2123-2132. https://doi.org/10.1056/NEJMoa1705848

11. Tepper S, Ashina M, Reuter U, Brandes JL, Doležil D, Silberstein S et al (2017) Safety and efficacy of erenumab for preventive treatment of chronic migraine: a randomised, double-blind, placebo-controlled phase 2 trial. Lancet Neurol 16(6):425-434. https://doi.org/10.1016/s1474-4422(17)30083-2

12. Reuter U, Goadsby PJ, Lanteri-Minet M, Wen S, Hours-Zesiger P, Ferrari MD et al (2018) Efficacy and tolerability of erenumab in patients with episodic migraine in whom two-to-four previous preventive treatments were unsuccessful: a randomised, double-blind, placebo-controlled, phase 3b study. Lancet 392(10161):2280-2287. https://doi.org/10.1016/s0140-6736(1 8) $32534-0$

13. Barbanti P, Aurilia C, Egeo G, Fofi L (2019) Erenumab: from scientific evidence to clinical practice-the first Italian real-life data. Neurol Sci 40(Suppl 1):177-179. https://doi.org/10.1007/s10072-019-03839-x

14. Ornello R, Casalena A, Frattale I, Gabriele A, Affaitati G, Giamberardino MA et al (2020) Real-life data on the efficacy and safety of erenumab in the Abruzzo region, central Italy. J Headache Pain 21(1):32. https://doi.org/10.11 86/s10194-020-01102-9

15. Raffaelli B, Kalantzis R, Mecklenburg J, Overeem LH, Neeb L, Gendolla A et al (2020) Erenumab in chronic migraine patients who previously failed five first-line oral prophylactics and onabotulinumtoxinA: a dual-center retrospective observational study. Front Neurol 11:417. https://doi.org/10.33 89/fneur.2020.00417

16. Russo A, Silvestro M, Scotto di Clemente F, Trojsi F, Bisecco A, Bonavita S et al (2020) Multidimensional assessment of the effects of erenumab in chronic migraine patients with previous unsuccessful preventive treatments: a comprehensive real-world experience. J Headache Pain 21(1):69. https:// doi.org/10.1186/s10194-020-01143-0

17. Lambru G, Hill B, Murphy M, Tylova I, Andreou AP (2020) A prospective realworld analysis of erenumab in refractory chronic migraine. J Headache Pain 21(1):61. https://doi.org/10.1186/s10194-020-01127-0

18. Scheffler A, Messel O, Wurthmann S, Nsaka M, Kleinschnitz C, Glas M et al (2020) Erenumab in highly therapy-refractory migraine patients: first German real-world evidence. J Headache Pain 21(1):84. https://doi.org/10.1186/s101 94-020-01151-0

19. Pensato U, Favoni V, Pascazio A, Benini M, Asioli GM, Merli E et al (2020) Erenumab efficacy in highly resistant chronic migraine: a real-life study. Neurol Sci. https://doi.org/10.1007/s10072-020-04658-1

20. Ranieri A, Alfieri G, Napolitano M, Servillo G, Candelaresi P, Di lorio W et al (2020) One year experience with erenumab: real-life data in 30 consecutive patients. Neurol Sci. https://doi.org/10.1007/s10072-020-04677-y

21. Talbot J, Stuckey R, Crawford L, Weatherby S, Mullin S (2021) Improvements in pain, medication use and quality of life in onabotulinumtoxinA-resistant chronic migraine patients following erenumab treatment - real world outcomes. J Headache Pain 22(1):5. https://doi.org/10.1186/s10194-02001214-2

22. Cheng S, Jenkins B, Limberg N, Hutton E (2020) Erenumab in chronic migraine: an Australian experience. Headache 60(10):2555-2562. https://doi. org/10.1111/head.13968

23. Disco C, Billo G, De Boni A, De Luca C, Perini F (2020) Efficacy of erenumab $70 \mathrm{mg}$ in chronic migraine: Vicenza experience. Neurol Sci 41 (Suppl 2):479480. https://doi.org/10.1007/s10072-020-04665-2

24. Robblee J, Devick KL, Mendez N, Potter J, Slonaker J, Starling AJ (2020) Realworld patient experience with erenumab for the preventive treatment of migraine. Headache 60(9):2014-2025. https://doi.org/10.1111/head.13951

25. Schiano di Cola F, Rao R, Caratozzolo S, Di Cesare M, Venturelli E, Balducci U et al (2020) Erenumab efficacy in chronic migraine and medication overuse: a real-life multicentric Italian observational study. Neurol Sci 41(Suppl 2): 489-490. https://doi.org/10.1007/s10072-020-04670-5

26. Matteo E, Favoni V, Pascazio A, Pensato U, Benini M, Asioli GM et al (2020) Erenumab in 159 high frequency and chronic migraine patients: real-life results from the Bologna Headache Center. Neurol Sci 41(Suppl 2):483-484. https://doi.org/10.1007/s10072-020-04667-0

27. Blumenfeld AM, Frishberg BM, Schim JD, lannone A, Schneider G, Yedigarova $L$ et al (2021) Real-world evidence for control of chronic migraine patients receiving CGRP monoclonal antibody therapy added to onabotulinumtoxinA: a retrospective chart review. Pain Ther. https://doi. org/10.1007/s40122-021-00264-x

28. Fang J, Korrer S, Johnson JC, Cheadle MA, Shah R, Ferraris ML et al (2021) Real-world trends in characteristics of patients with migraine newly initiated on erenumab in the USA: a retrospective analysis. Adv Ther 38(6):29212934. https://doi.org/10.1007/s12325-021-01677-y

29. Kanaan S, Hettie G, Loder E, Burch R (2020) Real-world effectiveness and tolerability of erenumab: a retrospective cohort study. Cephalalgia 40(13): 1511-1522. https://doi.org/10.1177/0333102420946725

30. Ornello R, Casalena A, Frattale I, Caponnetto V, Gabriele A, Affaitati G et al (2020) Conversion from chronic to episodic migraine in patients treated with erenumab: real-life data from an Italian region. J Headache Pain 21(1): 102. https://doi.org/10.1186/s10194-020-01171-w 
31. Torres-Ferrús M, Gallardo VJ, Alpuente A, Caronna E, Gine-Cipres E, PozoRosich P (2021) The impact of anti-CGRP monoclonal antibodies in resistant migraine patients: a real-world evidence observational study. J Neurol. https://doi.org/10.1007/s00415-021-10523-8

32. German Federal Joint Committee (Gemeinsamer Bundesausschuss=GBA). Erenumab: resolution and justification. Available online at: https://www.g-ba. de/bewertungsverfahren/nutzenbewertung/411/\#english. Assessed 19 Feb 2021

33. Report of the Association of Private Health Insurers in Germany. Avaiable online at https://www.pkv.de/fileadmin/user_upload/PKV/c_Verband/PDF/2 020-12_PKV-Zahlenbericht_2019.pdf. Assessed 19 Feb 2021

34. Diener H-C, Holle-Lee D, Nägel S, Dresler T, Gaul C, Göbel H et al (2019) Treatment of migraine attacks and prevention of migraine: guidelines by the German Migraine and Headache Society and the German Society of Neurology. Clin Transl Neurosci 3(1). https://doi.org/10.1177/2514183x 8823377

35. German Society of Neurology (Deutsche Gesellschaft Neurologie = DGP). Addendum to guideline 030/057 therapy of migraine attacks and prophylaxis. Available at: https://www.awmf.org/fileadmin/user_upload/ Leitlinien/030_D_Ges_fuer_Neurologie/030-057addendum_S1_MigraeneTherapie-Prophylaxe_2020-02-verlaengert.pdf.Assessed 19 Feb 2021

36. Ornello R, Tiseo C, Frattale I, Perrotta G, Marini C, Pistoia F et al (2019) The appropriate dosing of erenumab for migraine prevention after multiple preventive treatment failures: a critical appraisal. J Headache Pain 20(1):99. https://doi.org/10.1186/s10194-019-1054-4

37. Tepper SJ, Ashina M, Reuter U, Brandes JL, Doležil D, Silberstein SD et al (2020) Long-term safety and efficacy of erenumab in patients with chronic migraine: results from a 52-week, open-label extension study. Cephalalgia 40(6):543-553. https://doi.org/10.1177/0333102420912726

38. Schwedt T, Reuter U, Tepper S, Ashina M, Kudrow D, Broessner G et al (2018) Early onset of efficacy with erenumab in patients with episodic and chronic migraine. J Headache Pain 19(1):92. https://doi.org/10.1186/s10194018-0923-6

39. Volpicelli LK, Robertson C, Bhowmick A, Herbert LB (2020) Perceived treatment satisfaction and effectiveness facilitators among patients with chronic health conditions: a self-reported survey. Interact J Med Res 9(1): e13029. https://doi.org/10.2196/13029

40. Ament M, Day K, Stauffer VL, Skljarevski V, Rettiganti M, Pearlman E et al (2021) Effect of galcanezumab on severity and symptoms of migraine in phase 3 trials in patients with episodic or chronic migraine. J Headache Pain 22(1):6. https://doi.org/10.1186/s10194-021-01215-9

41. Martelletti P, Schwedt TJ, Lanteri-Minet M, Quintana R, Carboni V, Diener HC et al (2018) My migraine voice survey: a global study of disease burden among individuals with migraine for whom preventive treatments have failed. J Headache Pain 19(1):115. https://doi.org/10.1186/s10194-018-0946-z

42. Guidotti M, Ravasio R (2009) Clinical and economic comparison of frovatriptan versus other oral triptans in the treatment of acute migraine in the real-world setting. Clin Drug Investig 29(11):693-702. https://doi.org/1 0.2165/11315330-000000000-00000

\section{Publisher's Note}

Springer Nature remains neutral with regard to jurisdictional claims in published maps and institutional affiliations.

Ready to submit your research? Choose BMC and benefit from:

- fast, convenient online submission

- thorough peer review by experienced researchers in your field

- rapid publication on acceptance

- support for research data, including large and complex data types

- gold Open Access which fosters wider collaboration and increased citations

- maximum visibility for your research: over $100 \mathrm{M}$ website views per year

At BMC, research is always in progress.

Learn more biomedcentral.com/submissions 